\title{
Monitoring Trends of Self-diagnosis in New York City Emergency Departments
}

\author{
Alyssa Z. Chase* \\ Syndromic Surveillance, NYC Department of Health, Long Island City, New York, USA
}

\section{Objective}

To monitor self-reported diagnosis from New York City (NYC) emergency department (ED) chief complaints (CC).

\section{Introduction}

The number of US adults who use the internet to access health information has increased from about 95 million in $2005^{1}$ to 220 million in $2014^{2,3}$. The public health impact of this trend is unknown; in theory, patients may be able to better help the doctor arrive at the correct diagnosis, but self-diagnosed patients may also inappropriately self-treat or delay going to the doctor. The current study examines trends in self-diagnoses in NYC EDs, identifies the demographic characteristics of self-diagnosed patients, and compares hospital admission rates of self-diagnosed patients with those who do not self-diagnose.

\section{Methods}

The NYC syndromic surveillance system collects near real-time electronic data from 49 NYC EDs. The data include patient CC, which is entered using either a pick list, free text, or both. To identify cases of self-reported diagnosis, we scanned CCs for variations of the words "think," "believe," "suspect," "state," "say" in combination with the words "has" "have" or "having," excluding complaints where patients noted a non-specific disease, such as "infection" or "rash." In addition, we looked for the phrase "As per patient" and a specific diagnosis. To identify specific diagnoses, we limited our search to non-chronic diseases, which we hypothesized were more likely to have been previously diagnosed by a physician than an infectious illness or a major health event like appendicitis or stroke.

We monitored changes in the proportion of ED visits with selfdiagnoses from January 2003 to August 2014, sub-setting to ED visits that only included patient language in the CC. CCs with patient language were identified using the methods described above, and CCs that contained quotation marks or pronouns were also included.

Chi-square tests were used to measure associations between the rate of self-diagnoses and gender, age, and hospital admission status. Admission rates were used as a proxy for severity of patient illness and were only examined from January 2010 to August 2014 due to the high rate of missing admission values before this period.

\section{Results}

We found that $2.6 \%(n=1,2031,380)$ of all ED visits from 20032014 contained patient language in the CC. Within the patient language-only subset, the percentage of CCs containing a selfdiagnosis nearly doubled from 2004 to 2014, from 3\% to almost $6 \%$.

Patient demographics and admission rates were examined and found to be fairly consistent over time. Females self-reported a diagnosis more frequently than males $(54.6 \%$ versus $45.4 \%$, $\mathrm{p}<0.001)$. The most prominent differences between self-diagnosing and non-self-diagnosing patients were observed for patients 19-29 years $(28.0 \%$ versus $20.6 \%)$ and adults $65+$ years $(7.1 \%$ versus $10.6 \%$ ). Self-diagnosis was also associated with lower risk of hospital admission (9.5\% versus $10.4 \%, \mathrm{p}<0.001)$.

\section{Conclusions}

NYC EDs have seen an increasing trend in self-diagnosis from 2003-2014 consistent with nation-wide increases in internet usage for health information during the same period. Women and people aged 19-29 were most likely to self-diagnose, while patients aged 65+ were least likely to do so. These results are consistent with the results of a recent Pew Research Survey that examined self-diagnosis using the internet 4 . We also found that individuals who self-diagnosed were less likely to be admitted to the hospital, suggesting that self-diagnosis may have a positive health impact. Going forward, we will assess the accuracy of self-diagnosis by comparing patient self-diagnoses with clinician-assigned diagnoses available in hospitalization data from the Statewide Planning and Research Cooperative System of the New York State Department of Health.

\section{Keywords}

syndromic surveillance; self-diagnosis; Chief Complaint

\section{References}

1. Fox, Susannah. "Health Information Online." Pew Research Center, Washington D.C. (May 17, 2005). http://www.pewinternet. org/2005/05/17/health-information-online/, accessed on August 20, 2014.

2. Pew Research Center, Washington D.C. (2014). http://www. pewinternet.org/data-trend/internet-use/latest-stats/, accessed on August 20, 2014.

3. US Census Bureau. "Census Bureau Projects U.S. Population of 317.3 Million on New Year's Day" (December 30, 2013). http://www. census.gov/newsroom/releases/archives/population/cb13-tps112.html, accessed on August 20, 2014.

\footnotetext{
*Alyssa Z. Chase

E-mail: achase@health.nyc.gov
} 